\author{
Martine Remy-Jardin \\ Isabelle Tillie-Leblond \\ David Szapiro \\ Benoit Ghaye \\ Laurent Cotte \\ Ioana Mastora \\ Valérie Delannoy \\ Jacques Remy
}

\section{CT angiography of pulmonary embolism in patients with underlying respiratory disease: impact of multislice CT on image quality and negative predictive value}

Received: 8 November 2001

Revised: 29 March 2002

Accepted: 2 April 2002

Published online: 26 June 2002

(C) Springer-Verlag 2002

M. Remy-Jardin ( $)$ D. Szapiro

B. Ghaye · I. Mastora · J. Remy

Department of Radiology,

Hospital Calmette,

University Center of Lille,

Boulevard Jules Leclerc,

59037 Lille Cedex, France

e-mail: mremy-jardin@ chru-lille.fr

Tel.: +33-3-20444311

Fax: +33-3-20444720

M. Remy-Jardin · I. Mastora · J. Remy

Medical Research Group

"Equipe d'Accueil no. 2682",

Boulevard Jules Leclerc,

59037 Lille Cedex, France

I. Tillie-Leblond · L. Cotte

Department of Pulmonology,

Hospital Calmette,

University Center of Lille,

Boulevard Jules Leclerc,

59037 Lille Cedex, France

V. Delannoy

Department of Medical Statistics, University of Lille, Place de Verdun, 59037 Lille Cedex, France

\begin{abstract}
Our objective was to evaluate the impact of multislice CT (MSCT) on image quality and diagnostic value of spiral CT angiograms. Over an 8-month period (January 2000 to August 2000), 134 consecutive patients, including 55 patients with underlying lung disease, underwent MSCT (group 1). Image quality and diagnostic results of CT angiograms were compared with those obtained in 125 consecutive patients, including 58 patients with underlying lung disease, evaluated with thin-collimation single slice CT (SSCT; group 2) over a similar period of time (January 1999 to August 1999). A 3-month clinical follow-up was systematically obtained in all patients who were not anticoagulated in the two groups. For a significantly longer mean $\mathrm{z}$-axis coverage, the mean duration of data acquisition was significantly shorter with MSCT. The frequency of examinations devoid of motion artifacts was significantly higher in group 1 than in group 2. In the ab-
\end{abstract}

sence of significant difference in the quality of vascular enhancement, mainly coded as good or excellent, the proportion of examinations interpretable down to the subsegmental arteries was higher in group $1(57.5 \%)$ than in group $2(13 \%)(p<0.0001)$. The benefits of MSCT were more marked for patients with underlying respiratory disease and did not lead to a higher detection rate of peripheral pulmonary embolism. The negative predictive values of single-slice and multislice CT were 100 and $99 \%$, respectively. Improvement in image quality on MSCT scans accounts for the improved diagnostic accuracy of CT angiography, in particular for patients with impaired respiratory function.

Keywords Multislice CT . CT angiography $\cdot$ Pulmonary embolism - Pulmonary arteries . Chronic obstructive lung disease

\section{Introduction}

The introduction of spiral CT has considerably modified the diagnostic approach of acute pulmonary embolism (PE) and its accuracy has progressively improved in parallel to the improvement of CT technology over the past 10 years. Initially performed with single-slice CT scanners and a 5-mm collimation, CT angiography provided an accurate evaluation of central pulmonary arteries but was unable to reliably identify endoluminal clots within segmental and subsegmental branches [1, 2, 3, 4]. An important step was the availability of subsecond scanning in the late 1990s which allowed scanning of patients with thinner collimations, i.e., 3- to $2 \mathrm{~mm}$, over a longer region of interest. Whereas this optimization of technical factors allowed marked improvement in the evaluation of the segmental pulmonary arterial bed, there remained limitations in the evaluation of subsegmental 
pulmonary arteries $[5,6]$. With a recent increase in gantry speed from one to two revolutions per second, multisection CT scanners are now up to eight times faster than conventional single-section spiral CT scanners, allowing greater anatomic coverage and better longitudinal resolution during a single breath hold [7]. In a preliminary anatomical study, Ghaye et al. have shown that peripheral pulmonary arteries down to the fifth order were accurately analyzed on multislice spiral CT angiograms [8]. The purpose of the present study was to test the hypothesis that the increased scanning efficiency of four-section CT compared with one-section CT would improve CT angiography of the pulmonary circulation in clinical practice, in particular in the subset of patients with underlying respiratory disease.

\section{Materials and methods}

\section{Study population}

Between January 2000 and August 2000, 134 consecutive patients suspected of acute PE, including 55 patients with underlying respiratory disease, were referred from the Department of Pulmonology of our institution to the Department of Thoracic Imaging for spiral CT angiography of the pulmonary circulation using multislice CT (MSCT). This population, hereafter referred to as group 1, included 79 men and 55 women. For the purpose of evaluating the impact of MSCT in the diagnostic work-up of PE, this population was compared with that referred for suspicion of acute PE from the same pulmonology department over a similar period of time (January 1999 to August 1999) who underwent single-slice spiral CT angiography of the pulmonary circulation. This second population, hereafter referred to as group 2, included 125 patients ( 80 men, 45 women) among whom 58 had underlying respiratory disease. This study design was imposed by the availability of a single CT unit in the Department of Thoracic Imaging, consisting of a single-slice spiral CT scanner in the year 1999 (Somatom Plus 4A, Siemens, Forcheim, Germany), replaced by a multislice CT unit in January 2000 (Volume Zoom, Siemens, Forcheim, Germany).

All patients underwent CT angiography of the pulmonary circulation, indicated as the first-line diagnostic test for acute PE in our institution. The clinicians were free to order additional examinations, namely ventilation-perfusion scanning, color Doppler and venous compression ultrasonography (US), and/or pulmonary angiography at the time of the initial diagnosis. When spiral CT revealed negative findings, the patients who did not receive anticoagulation were followed-up for 3 months to detect any clinical events that could be related to PE. If PE was diagnosed during follow-up, the initial spiral CT study was labeled as false-negative.

\section{Spiral CT angiography}

In group 1, all spiral CT angiograms were obtained with a $4 \times 1-\mathrm{mm}$ collimation over the entire thorax, a table feed of $8 \mathrm{~mm}$ per rotation (pitch of 2.0), and a rotation time of $0.5 \mathrm{~s}$, leading to the reconstruction of 1.25 -mm-thick mediastinal and lung CT scans, obtained at 1-mm intervals. Owing to the number of images generated for each examination, four sets of images were printed on films:

1. Thin-collimated lung and mediastinal images, obtained at 10-mm intervals, allowing analysis of the entire thorax (field of view: $350 \mathrm{~mm}$ )
2. Thin-collimated lung and mediastinal images of the pulmonary circulation, obtained at 3-mm intervals, from the level of the subsegmental vessels of the upper lobes to the level of the subsegmental vessels of the lower lobes (field of view: $250 \mathrm{~mm}$ ), aimed at detecting endoluminal clots. In group 2, thin-collimated CT angiograms were obtained either with a 2-mm collimation and a pitch of $2.0(n=110)$ or a $3-\mathrm{mm}$ collimation and a pitch of $1.7(n=15)$ according to the patients' breath-hold capabilities (scanning time: $0.75-\mathrm{s}$ per revolution). The $\mathrm{z}$-axis coverage started at the level of the subsegmental vessels of the upper lobes down to the level of the subsegmental vessels of the lower lobes, both levels selected on the preconstrats CT scan further detailed. Contiguous transverse CT scans were systematically reconstructed at lung and mediastinal window settings using a field of view of $250 \mathrm{~mm}$. A CT angiography was systematically carried out by a noncontrast CT examination of the entire thorax, consisting of 1-mm thick transverse CT scans, obtained at $10-\mathrm{mm}$ intervals, reconstructed at lung and mediastinal window settings. All examinations were obtained in the craniocaudal direction. In both groups, the patients received an injection of $120-140 \mathrm{ml}$ of a $24-30 \%$ contrast agent, respectively, administered at a rate of 4 and $3 \mathrm{ml} / \mathrm{s}$ according to the venous access available. The start delay was selected according to the patients' hemodynamic characteristics, varying between 12 and $15 \mathrm{~s}$ for patients with normal hemodynamic status and between 18 and $20 \mathrm{~s}$ for patients suspected of pulmonary hypertension and/or right heart failure.

\section{Interpretation of spiral CT angiograms}

All CT examinations were prospectively read during the course of clinical work-up by subspecialty-trained chest radiologists, with the objective of assessing or excluding acute PE. For the purpose of the present study, these examinations were reinterpreted by consensus between two faculty radiologists (B.G., D.S.), both experienced in evaluating spiral CT studies for acute PE. Their objective was not only to evaluate the presence of acute PE, but also to grade image quality. Because of obvious differences in the number of images generated in group 1 and group 2, the observers were not blinded to the differences in CT technology; however, they were not aware of the patients' history nor clinical symptoms at the time of CT angiography.

Evaluation of image quality included analysis of the degree of vascular enhancement and the search for motion artifacts. For each angiogram, the overall degree of vascular enhancement was graded as excellent, good, moderate, or poor. In addition, the presence of a gradient of arterial enhancement from top to bottom of the region of interest was visually assessed by comparing the attenuation in A1 and in A10 (according to the nomenclature of the pulmonary arterial branches [9]). Two categories of motion artifacts were searched for on lung images, i.e., respiratory and cardiac motion artifacts, graded as absent (grade 0), minimal (grade 1), or altering the interpretation of images (grade 2). The predominant or exclusive location of artifacts in the upper, mid, or lower lung zones was systematically recorded. The criteria for motion artifacts included:

1. Double-imaged lung structures (bronchial walls, vessels, fissures)

2. Doubling and blurring of the cardiac border

3 Distortion of vessels resulting in star artifacts or low-attenuating areas adjacent to vessels

4. Linear streak artifacts in the lung parenchyma

When seen throughout both lungs on successive scans, the artifacts were classified as respiratory motion artifacts; when exclusively located in the paracardiac lung segments, the artifacts were considered to be due to transmitted cardiac motion. 
With regard to the diagnosis of acute PE, we considered the following scheme of interpretation. The presence of endoluminal clots on CT scans were considered diagnostic of embolism; central emboli included thrombi within main arteries, lobar arteries, or both; peripheral thrombi consisted of endoluminal clots within segmental and/or subsegmental branches. According to the technical quality of spiral CT scans, two levels of confidence in excluding PE were systematically considered. Spiral CT angiograms were considered negative down to the subsegmental level when a meticulous analysis of central and peripheral pulmonary arteries was possible due to an excellent degree of arterial enhancement from top to bottom of the region of interest on images devoid of respiratory motion artifacts. When suboptimal arterial enhancement and/or presence of motion artifacts was observed on part of the examination, confident depiction of subsegmental clots was not possible; therefore, in the absence of endoluminal clots within central and segmental arteries on such examinations, spiral CT scans were considered as negative down to the segmental level. When confident exclusion of PE on spiral CT scans was limited to central arteries, spiral CT was interpreted as inconclusive.

\section{Statistical analysis}

Statistical analysis was performed with commercially available software (SAS Institute, Cary, N.C.). Comparison of frequencies were carried out with the chi-square test or the Fisher exact test when tables had expected values of less than five. Comparison between two groups were evaluated using the Student's $t$-test $(n>30)$ or the Wilcoxon's signed-rank test $(n<30)$.

\section{Results}

Clinical characteristics of group-1

and group-2 populations

A summary of the clinical characteristics of group-1 and group-2 patients is provided in Table 1. Apart from the mean $( \pm \mathrm{SD})$ age of the patients who was higher in group 1 than in group 2, there was no significant difference in the gender ratio or in the referral location between the two groups of patients. No significant difference was found in the proportion of patients with underlying respi- ratory disease between group $1(n=55,41 \%)$ and group 2 $(n=58,46 \%)(p=0.12)$. Underlying lung disease consisted mainly of chronic obstructive pulmonary disease (COPD) in both groups of patients (group 1: 48 of 55, 87\%; group 2: 52 of 58, 90\%), whereas 7 patients in group 1 and 6 patients in group 2 presented miscellaneous lung disorders [group 1: complicated coal worker's pneumoconiosis $(n=3)$, sarcoidosis $(n=2)$, and idiopathic pulmonary fibrosis $(n=2)$; group 2: complicated coal worker's pneumoconiosis $(n=3)$, allergic bronchopulmonary aspergillosis $(n=2)$, and idiopathic pulmonary fibrosis $(n=1)]$. Severity of COPD was assessed according to the ATS criteria [10], considering three grades according to the value of forced expiratory volume in $1 \mathrm{~s}$ (FEV1), expressed as a percentage of predicted values: grade I, FEV1>50\%; grade II, FEV1 comprised between 35 and $50 \%$; and grade III, FEV1<35\%. In the study group, severity of COPD was as follows:

1. The 48 patients with COPD in group 1 comprised 17 $(35 \%)$ patients with a grade I, 14 patients $(30 \%)$ with a grade II $(n=14 ; \%)$, and 17 patients $(35 \%)$ with grade III.

2. The 52 patients with COPD in group 2 included 22 patients $(42 \%)$ with grade I, 17 patients $(33 \%)$ with grade II, and 13 patients (25\%) with grade III. Table 2 summarizes the investigations obtained in addition to spiral CT angiography in groups 1 and 2 .

Comparison of image quality between group 1 and group 2

Quality of vascular opacification did not significantly differ between the two groups as shown in Table 3 . Whereas the mean $( \pm \mathrm{SD})$ volume of contrast material administered was significantly lower in group 1 than in group 2 (126.45 \pm 14 vs $142.8 \pm 11 \mathrm{ml} ; p=0.0001)$, a constant degree of arterial enhancement from top to bottom

Table 1 Characteristics of the populations referred for spiral CT angiography

\begin{tabular}{llll}
\hline & Group 1 $(n=134)$ multislice CT & Group 2 $(n=125)$ single-slice CT & $p$-value \\
\hline Age, mean ( \pm SD; years) & $64 \pm 14$ & $59 \pm 17$ & 0.02 \\
Gender ratio (men/women) & $79(59 \%)$ men/55 $(41 \%)$ women & $80(64 \%)$ men/45 (36\%) women & n.s. \\
Referral location & & & \\
Outpatients & $23(17)$ & $25(20)$ & - \\
Inpatients & $57(42.5)$ & $61(49)$ & n.s. \\
Intensive care unit & $54(40)$ & $39(31)$ & n.s. \\
Presence of underlying respiratory disease & $55(41)$ & 58 of 58 & - \\
Chronic obstructive pulmonary disease & 48 of 55 & 6 of 58 & - \\
Miscellaneous causes & 7 of 55 & & \\
Risk factors & & $1.2 \pm 0.1$ & $\mathrm{~ns}$ \\
Average no. of risk factors per patient & $1 \pm 0.09$ & & \\
\hline
\end{tabular}

Numbers in parentheses are percentages 
Table 2 Results of investigations obtained in addition to spiral CT angiography in the overall population

Numbers in parentheses are percentages

All $p$-values were statistically insignificant

Table 3 Quality of arterial opacification on CT angiograms

Numbers in parentheses are percentages

\begin{tabular}{lcc}
\hline & $\begin{array}{c}\text { Group 1 }(n=134) \\
\text { multislice CT }\end{array}$ & $\begin{array}{l}\text { Group 2 }(n=125) \\
\text { single-slice CT }\end{array}$ \\
\hline Ventilation-perfusion scintigraphy & $28(21)$ & $17(13.6)$ \\
Normal & $9(6.7)$ & $6(4.8)$ \\
Low probability & $13(9.7)$ & $8(6.4)$ \\
Intermediate probability & $3(2.2)$ & $2(1.6)$ \\
High probability & $3(2.2)$ & $1(0.8)$ \\
Color Doppler ultrasound examination & $124(92.5)$ & $118(94.4)$ \\
Pulmonary angiography & $1(0.7)$ & 0 \\
\hline
\end{tabular}

\begin{tabular}{lcll}
\hline & $\begin{array}{l}\text { Group 1 }(n=134) \\
\text { multislice CT }\end{array}$ & $\begin{array}{l}\text { Group 2 }(n=125) \\
\text { single-slice CT }\end{array}$ & $p$ value \\
\hline Degree of arterial enhancement & & $\mathrm{ns}$ \\
Excellent & $108(81)$ & $88(70)$ & - \\
Good & $18(13)$ & $21(17)$ & - \\
Moderate & $8(6)$ & $16(13)$ & - \\
Poor & 0 & 0 & \\
\hline
\end{tabular}

Table 4 Overall quality of CT angiograms in group 1 and group 2

\begin{tabular}{|c|c|c|c|}
\hline & $\begin{array}{l}\text { Group } 1(n=134) \\
\text { multislice CT }\end{array}$ & $\begin{array}{l}\text { Group } 2(n=125) \\
\text { single-slice CT }\end{array}$ & $p$-value \\
\hline Technical failures & 0 & 0 & - \\
\hline $\begin{array}{l}\text { CT angiograms technically adequate for depiction } \\
\text { of endoluminal clots down to segmental pulmonary } \\
\text { angiography branches }\end{array}$ & $50(37.3)$ & $96(76.8)$ & $<0.0001$ \\
\hline $\begin{array}{l}\text { CT angiograms technically adequate for depiction } \\
\text { of endoluminal clots down to subsegmental pulmonary } \\
\text { angiography branches }\end{array}$ & $77(57.5)$ & $16(13)$ & $<0.0001$ \\
\hline
\end{tabular}

Numbers in parentheses are percentages

of the region of interest was observed with a significantly higher frequency in group 1 than in group 2 (98 vs $89 \% ; p=0.01)$. The mean $( \pm \mathrm{SD})$ start delay was $18 \pm 2.6 \mathrm{~s}$ in group 1 and $16 \pm 2.5 \mathrm{~s}$ in group $2(p=0.0001)$.

For a significantly longer mean $( \pm \mathrm{SD})$ z-axis coverage (group 1: $152 \pm 34.5 \mathrm{~mm}$; group 2: $110 \pm 10.6 \mathrm{~mm}$; $p<0.001)$, the mean $( \pm \mathrm{SD})$ duration of data acquisition was significantly shorter in group 1 than in group 2 $(17 \pm 4.7$ vs $21 \pm 2.8 \mathrm{~s} ; p<.0001)$. The frequency of examinations devoid of respiratory motion artifacts was significantly higher in group $1(n=85,63 \%)$ than in group 2 $(n=56,45 \% ; p<0.001)$. When present, grade-1 (minimal) respiratory motion artifacts were observed with a significantly lower frequency in group $1(n=22,16 \%)$ than in group $2(n=34,27 \% ; p<0.05)$; no significant difference was found between the frequency of respiratory motion artifacts altering the interpretation of images (grade- 2 artifacts) between group $1(n=27,10 \%)$ and group $2(n=35$, $28 \%$ ). Whereas the frequency of respiratory motion arti- facts in the upper and mid lung zones did not differ between group 1 and group 2 (upper lung zones: group 1, $n=8,6 \%$; group $2, n=4,4 \%$; mid lung zones: group 1, $n=10,7.5 \%$; group $2, n=10,9 \%$ ), a significantly lower frequency of respiratory motion artifacts was found in group $1(n=41,23 \%)$ compared with group $2(n=54$, $43 \% ; p<0.0001)$. Images free of cardiac motion artifacts were seen with a higher-frequency in group $1 \quad(n=78$, $58 \%)$ than in group $2(n=51,41 \% ; p<0.001)$. When present, grade-1 (minimal) cardiac motion artifacts were observed with a significantly lower frequency in group 1 $(n=42,31 \%)$ than in group $2(n=67,54 \% ; p<0.05)$. No significant difference was found between the frequency of cardiac motion artifacts altering the interpretation of images (grade-2 artifacts) between group $1(n=14,10 \%)$ and group $2(n=7,6 \%)$. Interpolation artifacts were not detected in group-1 CT examinations.

The overall quality of CT angiograms in groups 1 and 2 is summarized in Table 4. A higher proportion of ex- 
Table 5 Comparison of image quality according to the CT technology used in patients with and without underlying respiratory disease

\begin{tabular}{|c|c|c|c|c|c|c|}
\hline & \multicolumn{3}{|c|}{ Patients without underlying respiratory disease } & \multicolumn{3}{|c|}{ Patients with underlying respiratory disease } \\
\hline & $\begin{array}{l}\text { Group 1a }(n=79) \\
\text { multislice CT }\end{array}$ & $\begin{array}{l}\text { Group 2a }(n=67) \\
\text { single-slice CT }\end{array}$ & $p$-value & $\begin{array}{l}\text { Group } 1 \mathrm{~b}(n=55) \\
\text { multislice CT }\end{array}$ & $\begin{array}{l}\text { Group 2b }(n=58) \\
\text { single-slice CT }\end{array}$ & $p$-value \\
\hline $\begin{array}{l}\text { CT angiograms with } \\
\text { an excellent or good } \\
\text { quality of vascular } \\
\text { opacification } n(\%)\end{array}$ & 75 (85) & $55(82)$ & n.s. & $51(93)$ & $54(93)$ & n.s. \\
\hline $\begin{array}{l}\mathrm{CT} \text { angiograms } \\
\text { without cardiac motion } \\
\text { artifacts } n(\%)\end{array}$ & $44(56)$ & $28(42)$ & n.s. & $34(62)$ & $23(40)$ & $<0.05$ \\
\hline $\begin{array}{l}\text { Accurate interpretation } \\
\text { of CT angiograms } \\
\text { down to the } \\
\text { subsegmental arteries } \\
n(\%)\end{array}$ & $51(65)$ & 7 (10) & $<0.0001$ & $26(47)$ & $9(15.5)$ & $<0.001$ \\
\hline
\end{tabular}

Numbers in parentheses are percentages

aminations interpreted as technically adequate down to the subsegmental level was found in group $1(57.5 \%)$ compared with group $2(13 \% ; p<0.0001)$. No significant difference was found in the frequency of inconclusive CT scans between group 1 and group 2 .

Comparison of image quality according to previous history of respiratory disease in group 1 and group 2

In order to determine whether a category of patients could experience a greater benefit from the use of multislice CT, we compared the subgroups of patients without underlying respiratory disease in group 1 and group 2, further referred to as group 1a and group 2a, and the subgroups of patients with underlying respiratory disease in group 1 and group 2, further referred to as group $1 \mathrm{~b}$ and group $2 \mathrm{~b}$. Among patients without previous history of respiratory disease, the only significant difference was a higher frequency of accurate interpretations of pulmonary arteries down to subsegmental branches on multislice CT angiograms compared with single-slice CT angiograms (65 vs 10\%; $p<0.0001$; (Table 5). Among patients with underlying respiratory disease, we observed significantly higher proportions of CT angiograms devoid of respiratory and cardiac motion artifacts as well as a significantly higher frequency of accurate interpretations down to the subsegmental arterial level on multislice CT angiograms (Table 5). The proportion of respiratory motion artifacts in the lower lung zones was significantly lower in group $1 \mathrm{~b}$ than in group $2 \mathrm{~b}(n=17,31 \%$ vs $n=30$, $58 \% ; p<0.01$ ). Minimal (grade-1) respiratory motion artifacts were seen with a significantly lower frequency in group $1 \mathrm{~b}(n=8,14.5 \%)$ compared with group $2 \mathrm{~b}(n=20$, $34 \% ; p<0.01)$, whereas the frequency of respiratory motion artifacts altering the interpretation of images did not differ between group $1 \mathrm{~b}(n=12,22 \%)$ and group $2 \mathrm{~b}$ $(n=16,28 \%)$. Cardiac motion artifacts were graded as minimal in 18 patients (33\%) in group $1 \mathrm{~b}$ and in 32 patients $(55 \% ; p<0.01)$, whereas the proportions of cardiac motion artifacts altering the interpretation of images did not significantly differ between groups $1 \mathrm{~b}$ and $2 \mathrm{~b}$, observed in $3(5 \%)$ patients in group $1 \mathrm{~b}$ and $3(5 \%)$ patients in group $2 b$.

Diagnostic accuracy of CT angiography in group 1 and group 2

Regarding the results of CT angiography in group 1 and group 2, no significant difference was found in the prevalence of acute PE whatever considering the entire study groups (group 1: $n=24,18 \%$; group 2: $n=27,22 \%$ ) or the subgroups of patients with and without underlying respiratory diseases (Table 6). No significant difference was found in the frequency of peripheral PE between group 1 and group 2 nor between the subgroups of patients with and without underlying respiratory disease scanned with multislice and single-slice CT (Table 6). A single case of isolated subsegmental PE, depicted in the apical segmental artery of the right upper lobe, was diagnosed in group $2 b$.

Among the 259 patients who underwent CT angiography, 43 patients in group 1 and 63 patients in group 2 were not considered in the follow-up study because of the following: 
Table 6 Spiral CT angiography results in patients with and without underlying respiratory disease in group 1 and group $2 . P E$ pulmonary embolism

\begin{tabular}{|c|c|c|c|c|}
\hline & \multicolumn{2}{|c|}{ Patients without underlying respiratory disease } & \multicolumn{2}{|c|}{ Patients with underlying respiratory disease } \\
\hline & $\begin{array}{l}\text { Group 1a }(n=79) \\
\text { multislice CT }\end{array}$ & $\begin{array}{l}\text { Group 2a }(n=67) \\
\text { single-slice CT }\end{array}$ & $\begin{array}{l}\text { Group } 1 \mathrm{~b}(n=55) \\
\text { multislice CT }\end{array}$ & $\begin{array}{l}\text { Group } 2 b(n=58) \\
\text { single-slice CT }\end{array}$ \\
\hline Absence of PE & $62(78.5)$ & $53(79)$ & $48(87)$ & $45(78)$ \\
\hline Presence of PE & $17(21.5)$ & $14(21)$ & $7(13)$ & $13(22)$ \\
\hline \multicolumn{5}{|l|}{ Extent of PE } \\
\hline Peripheral and/or central PE & $9(11)$ & $9(13)$ & $3(5)$ & $7(16)$ \\
\hline Segmental PE & $8(10)$ & $5(7.5)$ & $4(7)$ & $3(5)$ \\
\hline Isolated subsegmental PE & 0 & 0 & 0 & $1(2)$ \\
\hline
\end{tabular}

Numbers in parentheses are percentages

1. The presence of pulmonary embolism on CT scans (group 1, $n=24$; group 2, $n=27$ )

2. Anticoagulation for isolated venous thrombophlebitis (group 1,n=5; group 2,n=5) or cardiac arrhythmia (group 1, $n=1$; group 2, $n=2$ )

3. An inconclusive CT scan (group $1, n=7$; group 2, $n=13$ )

4. Because they were lost to follow-up (group 1, $n=6$ [4\%]; group 2, $n=6[5 \%])$

A 3-month clinical follow-up of the remaining 153 patients (group 1: $n=91$; group 2: $n=62$ ) led to the recognition of a single case of recurrent PE in group 1. This patient was an 84-year-old man with underlying COPD (grade-1 disease) who did not undergo ventilation-perfusion scintigraphy; CT angiography was negative down to the subsegmental pulmonary arteries. Despite positive findings on a sonographic examination of the limbs, this patient was not anticoagulated due to the concurrent presence of a left lung carcinoma in close contact with the left atrium and ipsilateral hilar pulmonary veins. The fatal event occurred 2 months after the CT angiographic examination. The negative predictive values of singleslice and multislice CT were 100 and 99\%, respectively.

\section{Discussion}

Our results suggest that improved quality of CT angiograms can be obtained with multislice CT in the clinical setting of suspected acute PE. Comparing two populations respectively scanned with multislice $\mathrm{CT}$ and singleslice CT, we intentionally focused on three parameters known to interfere with the overall image quality, namely the presence of respiratory and cardiac motion artifacts and the section collimation. Firstly, we observed a significantly higher proportion of examinations devoid of respiratory motion artifacts with multislice CT [85 (63\%) of 134 examinations] compared with single-slice CT [56 (36.5\%) of 125 examinations]. This improvement is likely to be related to the increase in scanning speed available on the multislice scanner, combining a shorter scanning time $(0.5 \mathrm{~s}$ per rotation vs $0.75 \mathrm{~s}$ with singleslice $\mathrm{CT}$ ) and the simultaneous acquisition of four slices per rotation. Consequently, the mean duration of data acquisition, and thus the mean breath-holding time, was significantly shorter for multislice spiral CT examinations (17 vs $21 \mathrm{~s}$ for single-slice CT angiograms). This improvement was observed together with the coverage of a longer region of interest as multislice spiral CT acquisitions systematically covered the entire thorax while single-slice spiral CT scans consisted of scanning a 10- to $12-\mathrm{cm}$ region of interest. Using the latter protocol, it is now possible to examine all the vasculature of interest on the angiograms, whereas the subsegmental arteries of the upper and/or lower lobes were not systematically examined with single-slice CT. With regard to the depiction of isolated subsegmental PE, it is important that CT systematically include these branches so that relevant PE are not missed, in particular for patients with impaired respiratory function.

Two categories of respiratory motion artifacts were observed in groups 1 and 2, those present throughout the examination because the patient was too dyspneic (and thus scanned while breathing), and those observed at the end of data acquisition due to the impossibility to maintain apnea until the end of data acquisition. The first situation can render single sections or an entire study nondiagnostic, whereas the second situation corresponds to altered images in the lower third of the volume scanned. Concerning the first category of artifacts, there was no significant difference between groups 1 and 2, as suggested by the comparison of respiratory motion artifacts present in the upper and mid lung zones; however, we found a significant reduction in the frequency of respiratory motion artifacts in the lower lung zones of multislice CT angiograms, observed in 31 (23\%) of 134 examinations compared with $54(43 \%)$ of 125 single-slice CT scans. As this anatomical region corresponds to the lower third of data acquisition due to the craniocaudal 
direction of the examinations in the present study, these results suggest that the shorter scanning time was more favorable for the maintain of strict apnea. As expected, the faster scanning capability also accounts for reduced unvoluntarily motion artifacts such as those resulting from the transmission of cardiac contractions to the surrounding lung parenchyma. We found that $58 \%$ of multislice CT scans (78 of 134 examinations) were free of cardiac motion artifacts compared with $41 \%$ of singleslice CT examinations (51 of 125 examinations). It is noteworthy that the rotation time of $500 \mathrm{~ms}$ currently available allows obtainment of images during the diastole of patients with a heart rate of 70 beats per minute; however, disturbing respiratory and cardiac motion artifacts were still observed in $27(20 \%)$ and $14(10 \%)$ of multislice CT angiograms, respectively. This finding is not surprising as Ritchie et al. have previously demonstrated that scanning times of less than $19.1 \mathrm{~ms}$ would be necessary for an effective suppression of motion artifacts on CT images [11]. Among the various methods proposed to correct scans containing motion, one can expect further improvement from the forthcoming generation of multislice scanners enabling the acquisition of more than four slices per rotation with shorter scanning times. In addition, the availability of retrospective ECG gating for spiral CT examinations of the entire thorax should help improve image quality, enabling reconstruction of images during the diastole of the cardiac cycle when ventricular movement is at its minimum [12].

The third benefit of multislice over single-slice CT is that the examinations can be performed with thinner sections, leading to higher spatial resolution along the longitudinal axis of the patient. In the present study, multislice CT examinations were acquired with a $4 \times 1-\mathrm{mm}$ collimation with reconstruction of $1.25-\mathrm{mm}$-thick transverse CT scans, whereas the effective section thicknesses of single-slice spiral CT scans acquired with a 2- to 3-mm collimation were 2.65 and $3.86 \mathrm{~mm}$, respectively. Reduction in scan collimation permits a decrease in the volumeaveraging effect between parenchyma, vessel walls, and blood, responsible for areas of low attenuation which may be confused with PE on transverse CT scans. Such partial identification of vessels may create false-positive and false-negative results or lead the observer to an indeterminate answer [13]. Using thin-collimation and subsecond single-slice CT, Remy-Jardin et al. have previously reported significant improvement in image quality due to a higher spatial resolution, resulting from a higher rate of analyzable segmental pulmonary arteries and a reduction in interpretive difficulties due to volume averaging $[5,6]$. Further improvement has recently been shown with multislice CT enabling an accurate analysis of peripheral pulmonary arteries down to the fifth order [8]. In the present study, an improved spatial resolution on images free of motion artifacts probably accounts for the significant increase in the proportion of examinations coded as technically adequate for an accurate interpretation of pulmonary arteries down to the subsegmental level (57.5 vs $13 \%$ with single-slice CT). In addition, it is emphasized that the interpolation artifacts were not detectable on multislice CT scans, confirming that a pitch of 2.0, and its inherent advantages in terms of breathhold duration, can be used for the evaluation of the pulmonary circulation in routine clinical practice.

This improvement in image quality was particularly noticeable in the subgroup of patients with underlying respiratory disease. Compared with single-slice $\mathrm{CT}$, and for a similar degree of functional impairment, we observed a significantly higher proportion of CT angiograms devoid of respiratory (64 vs 38\%) and cardiac motion artifacts (62 vs 40\%) with multislice CT. Because of the well-known difficulties in assessing acute PE in patients with underlying respiratory disease, the benefit of multislice CT, which allows obtainment of a higher number of examinations interpretable down to the subsegmental pulmonary arterial bed compared with single slice CT, is emphasized. As underlined by several investigators $[2,4,14,15]$, the limitations of single-slice CT in the detection of clots in small vessels are related mainly to lower spatial resolution and to the fact that subsegmental vessels of the upper and lower lobes frequently lie outside the limits of z-axis coverage. Consequently, the remaining indications of pulmonary angiography, namely the exclusion of subsegmental PE after a negative CT angiogram down to the segmental level, are expected to dramatically decline with the widespread availability of multislice CT scanners. Because improved image quality raised concerns about the detectability of small-sized clots with uncertain clinical significance, we paid particular attention to the prevalence of isolated subsegmental PE in group 1, in particular in the subgroup of patients with impaired lung function in whom isolated subsegmental clots have been shown to be responsible for acute clinical symptoms $[2,15]$. It is noteworthy that the improvement in spatial and temporal resolution observed in the present study did not lead to unusually high rates of positive angiograms in group 1, in particular of isolated subsegmental PE. The overall prevalence of acute PE was $18 \%$ in group 1 and $22 \%$ in group 2, otherwise lower than that reported in the PIOPED study [16]. In our opinion, this finding reflects the widespread acceptance of CT angiography by clinicians as a first-line minimally invasive diagnostic test for $\mathrm{PE}$ in clinical practice, more frequently indicated than pulmonary angiography. With regard to the diagnosis of isolated subsegmental PE, we diagnosed a single case in a group-1 patient with underlying COPD (one of 24 patients with positive angiograms, i.e., 4\%). Apart from Goodman et al. who identified four cases among 11 positive scans (36\%) [2], the reported prevalence of subsegmental PE on single-slice and dual-section spiral CT angiograms varies from 2 to $10 \%[3,4,6,15]$. 
These results illustrate our experience with multislice $\mathrm{CT}$ in the diagnostic work-up of PE. Our study design included the comparison of scans from different populations, a limitation inherent to the availability of a single CT unit at a time in our department; however, it should be pointed out that, apart a higher mean age of patients in group 1, we found no significant difference in the gender ratio, referral locations, and risk factors for PE between groups 1 and 2. Moreover, we observed a similar proportion of patients with and without underlying respiratory disease in both groups, due mainly to the presence of COPD. In addition, it is noteworthy that both series of CT angiograms did not differ in terms of quality of vascular enhancement, a parameter known to considerably influence the overall accuracy of CT in the depiction of acute PE; therefore, differences in image quality between group 1 and group 2 can be considered as reflecting technical differences between CT scans. From a practical standpoint, the analysis of peripheral pulmonary arteries on hard copies was judged time-consuming by the two readers, especially for the reconstructed scans of $1.25-\mathrm{mm}$-thick sections. Further studies are needed to establish whether these vessels can be accurately depicted alternatively with diagnostic workstations.

In conclusion, multislice CT is a technical improvement of spiral CT and a diagnostic tool of high sensitivity and specificity for the detection of PE. The improved spatial and temporal resolution benefited particularly the patients with impaired respiratory function for whom CT angiography now reaches the field of accuracy previously reported for nonrespiratory patients.

\section{References}

1. Remy-Jardin M, Remy J, Wattinne L, Giraud F (1992) Central pulmonary thromboembolism: diagnosis with spiral volumetric CT with the singlebreathhold technique: comparison with pulmonary angiography. Radiology 185:381-387

2. Goodman LR, Curtin JJ, Mewissen MW et al. (1995) Detection of pulmonary embolism in patients with unresolved clinical and scintigraphic diagnosis: helical CT versus angiography. Am J Roentgenol 164:1369-1374

3. Remy-Jardin M, Remy J, Deschildre F et al. (1996) Diagnosis of pulmonary embolism with spiral CT: comparison with pulmonary angiography and scintigraphy. Radiology 200:699-706

4. Van Rossum AB, Pattynama PM, Ton ER et al. (1996) Pulmonary embolism: validation of spiral CT angiography in 149 patients. Radiology 201:467-470

5. Remy-Jardin M, Remy J, Artaud D, Deschildre F, Duhamel A (1997) Peripheral pulmonary arteries: optimization of the spiral CT acquisition protocol. Radiology 204: 157-163
6. Remy-Jardin M, Remy J, Baghaie F, Fribourg M, Artaud D, Duhamel A (2000) Clinical value of thin-collimation in the diagnostic workup of pulmonary embolism. Am J Roentgenol 175:407-411

7. Rydberg J, Buckwalter KA, Caldermayer KS et al. (2000) Multisection CT: scanning techniques and clinical applications. Radiographics 20:17871806

8. Ghaye B, Szapiro D, Mastora I, Delannoy V, Duhamel A, Remy J, Remy-Jardin M (2001) Peripheral pulmonary arteries: How far in the lung does multidetector-row spiral CT allow analysis? Radiology 219:629-636

9. Boyden E (1955) Segmental anatomy of the lung. McGraw-Hill, New York

10. ATS statement (1995) Standards for the diagnosis and care of patients with chronic obstructive pulmonary disease. Am Rev Crit Care Med 152:578-583

11. Ritchie CJ, Godwin JD, Crawford CR, Stanford W, Anno H, Kim Y (1992) Minimum scan speeds for suppression of motion artifacts in CT. Radiology 185:37-42

12. Ohnesorge B, Flohr T, Becker C, Kopp AF, Schoepf UJ, Baum U, Knez A, Klingenbeck-Regn K, Reiser MF (2000) Cardiac imaging by means of electronically gated multisection spiral CT: initial experience. Radiology 217:564-571
13. Chatrand-Lefebvre C, Howarth N, Lucidarme O, Beigelman C, Cluzel Ph, Mourey-Gerosa I, Grenier P (1999) Contrast-enhanced helical CT for pulmonary embolism detection: inter- and intraobserver agreement among radiologists with variable experience. Am J Roentgenol 172:107-112

14. Garg K, Welsh CH, Feyerabend AJ, Subber SW, Russ PD, Johnston RJ, Durham JD, Lynch DA (1998) Pulmonary embolism: diagnosis with spiral $\mathrm{CT}$ and ventilation-perfusion scanning: correlation with pulmonary angiographic results or clinical outcome. Radiology 208:201-208

15. Quanadli SD, Hajjam ME, Mesurolle B, Barre O, Brucker F, Joseph T, Mignon F, Vieillard-Baron A, Dubourg O, Lacombe P (2000) Pulmonary embolism detection: prospective evaluation of dual-section helical CT versus selective pulmonary arteriography in 157 patients. Radiology 217:447-455

16. The PIOPED Investigators (1990) Value of the ventilation/perfusion scan in acute pulmonary embolism. J Am Med Assoc 263:2753-2759 